\title{
Design of Optimum Electromagnetic Absorbers in the Wireless Communications Range ${ }^{{ }^{*}}$
}

\section{Diseño de absorbedores electromagnéticos óptimos en el rango de las frecuencias inalámbricas ${ }^{2 *}$}

\author{
Edgar Eduardo Salazar Flórez \\ Julián Eduardo Mora Moreno ${ }^{4}$ \\ Carlos Rodrigo Correa Cely ${ }^{5}$
}

\footnotetext{
${ }^{1}$ Reception date: May 15th, 2012. Acceptance date: August 20th, 2013. This paper is based on a research project called Diseño de absorbedores electromagnéticos óptimos usando PSO y análisis de intervalos. It was developed by the research group CEMOS from Universidad Industrial de Santander, Bucaramanga, Colombia.

* The authors wish to thank Prof. Dr. Siedfreg M. Rump from the Institute for Reliable Computing at Hamburg, Germany, for allowing us to use the toolbox INTLAB, which was used for handling everything related to interval analysis.

${ }^{2}$ Fecha de recepción: 15 de mayo de 2012. Fecha de aceptación: 20 de agosto de 2013. Este artículo se basa en el proyecto de investigación denominado Diseño de absorbedores electromagnéticos óptimos usando PSO y análisis de intervalos. Fue desarrollado por el grupo de investigación CEMOS, de la Universidad Industrial de Santander, Bucaramanga, Colombia. ${ }^{3}$ Electronic engineer, Universidad Industrial de Santander, Bucaramanga, Colombia. E-mail: Salazar.edgar8@gmail.com. ${ }^{4}$ Electronic engineer, Universidad Industrial de Santander, Bucaramanga, Colombia. E-mail: juedmo@gmail.com.

${ }^{5}$ Chemical engineer, Universidad Nacional de Colombia. PhD, Lehigh University, Pennsylvania, USA. Professor Universidad Industrial de Santander, Bucaramanga, Colombia. E-mail: crcorrea@uis.edu.co.
} 


\section{$\Delta \nabla \nabla$}

This article shows the most relevant results, related to the optimum design of a multilayer electromagnetic absorber for the wireless communications range. It was designed through two optimization strategies, a metaheuristic (Particle Swarm Optimization [PSO]) and a deterministic one (Interval Analysis). Despite achieving similar results, the last one proved to require an increased amount of computation time. Nevertheless, and due to its nature, the solution achieved was unique, while in PSO the results reproducibility was low, possibly due to the high complexity of the objective function.

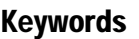

Electromagnetic absorber, optimization, particle swarm, interval analysis.

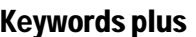

Electromagnetic wave absorber, optimization, particle swarm, Interval analysis (Mathematics).

\section{㳏以门米}

En este artículo se presentan los principales resultados asociados con el diseño óptimo de un absorbedor electromagnético plano multicapa, para el rango de frecuencias de las comunicaciones inalámbricas. Se diseñó mediante dos estrategias de optimización: una metaheurística (el algoritmo de enjambre de partículas) y otra determinística (el análisis de intervalos). A pesar de que se alcanzaron resultados similares, el método de intervalos mostró consistentemente un mayor tiempo de cómputo. Sin embargo y dada su naturaleza, su solución fue única, mientras que el método de enjambre de partículas mostró una limitada reproducibilidad en sus resultados, debido posiblemente al alto grado de complejidad de la función objetivo.

\section{2}

Absorbedor electromagnético, optimización, enjambre de partículas, análisis de intervalos.

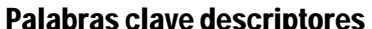

Absorbedor electromagnético, optimización, enjambre de partículas, análisis de intervalos (Matemáticas). 


\section{Introduction}

Electromagnetic absorbers are elements used to ideally attenuate all of the energy that impinges upon them. Because of this, they are essential in applications such as invisibility (at some given frequencies) and, especially, the construction of anechoic chambers, which are used by the telecommunication industry to perform electromagnetic compatibility tests (EMC) and measure, among others, the radiation parameters of antennas. Moreover, the evolution of wireless communications, as well as the non-conclusive reports on the effect of non-ionizing radiation on the human health, has transformed the design of electromagnetic absorbers into quite a demanding task (Vecchia et al., 2009). Its design, for any geometry (pyramid, multilayered, etc.) requires a balance between all the variables that affect its performance (Nornikman et al., 2012). Electromagnetic absorbers can be divided into two types: resonant absorbers and broadband absorbers. The first type relies on the material interacting with the incident radiation in a resonant way at a specific frequency. On the other hand, the broadband absorbers generally rely on materials whose properties are frequency independent and therefore can absorb radiation over a large bandwidth. One of the most widespread uses of electromagnetic absorbers is for radar cross section reduction, as well as, in reducing side lobe radiation in antennas. Watts et al. (2012), in a progress report, give a state of the art overview of the field of metamaterial electromagnetic wave absorbers and discuss a set of examples and related interesting applications. The physical dimensions and the nature of the materials play a big role (Zhao et al., 2013). They must be chosen in order to guarantee an acceptable attenuation for a given set of frequencies. Currently there are several numerical approaches that can be used to obtain the optimum point (traditionally performed by deterministic techniques which require knowing, among others, the Jacobian and the Hessian of the objective function). Ever since the boom of the heuristic techniques, a detailed knowledge of the objective function became barely relevant. Actually, two of the most known techniques for solving engineering problems are genetic algorithms and PSO (Cui et al., 2006; 
Afsar et al., 2011; Robinson and Rahmat-Samii, 2004; Cui and Weile, 2005; Chamaani et al., 2008; Michielssen et al., 1993; Liu et al., 2009).

In spite of their simplicity, ease of programming and low computation time, they present a drawback regarding repeatability, reproducibility and precision. This is even more relevant due to the complexity of the objective function. One alternative for this is to use, simultaneously, a deterministic algorithm. During this article, PSO and interval analysis were used for the absorber design. In the end, their advantages and disadvantages are compared, and the viability of using it for this case is shown.

\section{Fundamentals}

\subsection{Electromagnetic Absorbers}

An electromagnetic absorber is a device used to dissipate its incident energy. The main performance parameter is the reflection coefficient, which depends on the relative permittivity and permeability (often being complex values), and which are given by:

$$
\begin{aligned}
& \varepsilon_{r}=\varepsilon_{r}-j \varepsilon_{r} \tan \left(\delta_{d}\right) \\
& \mu_{r}=\mu_{r}-j \mu_{r} \tan \left(\delta_{m}\right)
\end{aligned}
$$

Where $\delta_{d}$ y $\delta_{m}$ are the electric and magnetic loss angles, respectively.

Figure 1 shows the general scheme for a multilayer electromagnetic absorber. Each layer is composed of a material with its own permittivity and permeability. The final one is in contact with a perfect conductor. Besides, it is assumed that the wave arrives perpendicularly.

The electric and magnetic fields for each region can be described as a function of the incident and reflected fields, as shown respectively in equations (3) and (4):

$$
\begin{aligned}
& E_{x}(z)=E_{i} e^{-\gamma z}\left[1+\frac{E_{r} e^{-2 \gamma z}}{E_{i}}\right] \\
& H_{y}(z)=E_{i} e^{-\gamma z}\left[1+\frac{E_{r} e^{-2 \gamma z}}{E_{i}}\right]
\end{aligned}
$$


Figure 1. Multilayer Absorber

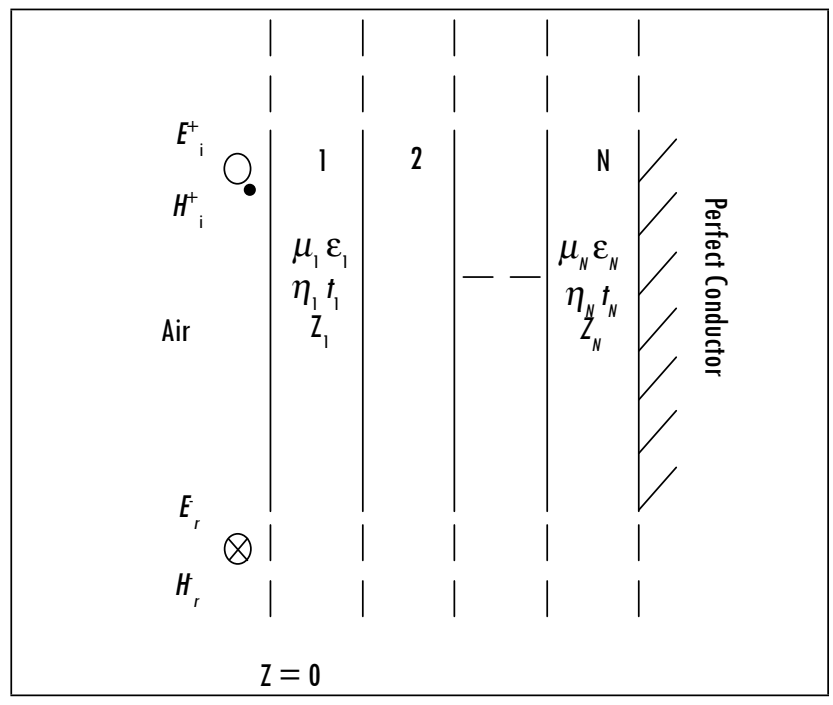

Source: Chamaani et al. (2008).

Where $z$ is a perpendicular axis that goes through the multilayered absorber, $\gamma=\sigma+j \beta$ and $\eta$ is the material's intrinsic impedance. On the other hand, the reflection coefficient is defined by:

$$
\Gamma(z)=\frac{E_{r} e^{2 \gamma z}}{E_{i}}
$$

Considering that the absorber's total field impedance is a relation of its electric and magnetic field, and by use of equations (3)-(5), the following relations can be established:

$$
\mathrm{Z}(z)=\eta \frac{1+\Gamma(z)}{1-\Gamma(z)}, \text { were } \mathrm{G}(z)=\frac{\mathrm{Z}(z)-\eta}{\mathrm{Z}(z)+\eta}
$$

According to transmission line theory, the total impedance of a material $k$, with $n$ layers, is given by (Bronwell, 1994):

$$
\mathrm{Z}_{k}=\left\{\begin{array}{c}
\eta_{k} \frac{\mathrm{Z}_{k+1}+\eta_{k} \tanh \left(\gamma_{k} t_{k}\right)}{\eta_{k}+\mathrm{Z}_{k+1} \tanh \left(\gamma_{k} t_{k}\right)} k<n \\
\eta_{n} \tanh \left(\gamma_{n} t_{n}\right) k=n
\end{array}\right.
$$


Where $t_{k}$ is the $k$-th layer thickness. The impedance of the last material $(k=n)$, is derived from its contact with the perfect conductor. In uhf applications $\gamma=j \beta$ (Bronwell, 1994), and equation (7) transforms into:

$$
\mathrm{Z}_{k}=\left\{\begin{array}{c}
\eta_{k} \frac{\mathrm{Z}_{k+1}+j \eta_{k} \tan \left(\beta_{k} t_{k}\right)}{\eta_{k}+j \mathrm{Z}_{k+1} \tan \left(\beta_{k} t_{k}\right)} k<n \\
j \eta_{n} \tan \left(\beta_{n} t_{n}\right) k=n
\end{array}\right.
$$

With $\beta_{\mathrm{k}}$ the $k$-th layer phase constant, which is defined as:

$$
\beta_{k}=2 \pi f \sqrt{\mu_{k} \varepsilon_{k}}
$$

The objective function for an electromagnetic absorber depends on the reflection coefficient, since it is a measure of the absorption efficiency. The total field impedance, $Z$ shown in equation (6), is transformed into the material 1 impedance, which is a function of the inner absorbing layers. The intrinsic impedance $\eta$ is the one from air, which can be approximated to the vacuum's. Therefore, it is rewritten as:

$$
R(f)=\frac{\mathrm{Z}_{1}(f)-\eta_{0}}{\mathrm{Z}_{1}(f)+\eta_{0}}
$$

Under these conditions, it is established that the design of the absorber must be done on a given set of frequencies. Thus, the objective function is proposed as (Zhao et al., 2013; Cui et al., 2006):

$$
F_{o b j}=20 \log (\max |R(f)|, f \in B)
$$

Where $B$ is the design band. Since the layer's thicknesses, as well as their electromagnetic properties, are unknown, whenever an N-layered electromagnetic absorber is designed, there will be $2 \mathrm{~N}$ parameters for the optimization.

\subsection{Interval Analysis}

Interval analysis is a mathematical method used to lock the solution for a given problem, described by equations. This analysis is based on a set of definitions, properties and operations that build a specific arithmetic. An interval, which is the basic unit, is defined as: 


$$
X=[a, b]=\{x \in \mathbb{R}: a \leq x \leq b\}
$$

An interval is described by two quantities, width $(X)$ and the middle point $\left(\operatorname{mid}\left(X_{1}\right)\right)$, defined as:

$$
\begin{aligned}
& \text { width }=(\operatorname{Sup}(X)-\operatorname{Inf}(X)) \\
& \operatorname{midth}\left(X_{1}\right)=\operatorname{Inf}(X)+\frac{\text { width }(X)}{2}
\end{aligned}
$$

The infimum $(\operatorname{Inf}(X))$ and supremum $(\operatorname{Sup}(X))$ of an interval refer to the lower and upper limits, respectively. Operating two or more intervals implies to build an answer interval, as shown in (15).

$$
X \cdot Y=\{x \cdot y: x \in \mathcal{E} X, y \in Y\}
$$

Definitions (12) and (15), even though simple, are the base of interval arithmetic. However, in order to identify the critical points of a function, it is necessary to deepen in the study of interval analysis. This implies knowing all the concepts related with the mapping of an expression in the real domain, into the interval one (isotonic inclusion, unity extension, interval extension, natural interval extension, fundamental theorem) (Moore, Baker, and Cloud, 2009).

Based on these concepts, it can be posed that by using interval variables in a function domain, the solution range would be an overestimate of the real one. This is the main obstacle, if it is desired to obtain a precise point. An appropriate way to deal with it, is to use Lipschitz definition: It is said that an interval extension, is Lipschitz on an interval $X_{0}$ if there is a constant $L$ such that:

$$
\omega(F(K)) \leq L \omega(K) \forall K \underline{\mathrm{C}} X_{0}
$$

This implies that the smaller the $K$, the more exact the output interval, $F$ $(K)$, will be. Therefore, it is possible to obtain a closer solution interval (ISR), by dividing $X_{0}$ into $\mathrm{N}$ parts, i.e.:

$$
I S R=\left(F\left(K_{1}\right) \underline{\cup} F\left(K_{2}\right) \underline{\cup} \cdots \underline{\cup} F\left(K_{N}\right)\right)
$$


Where $\bigcup$ represents the Hull interval. The previous process is known as refinement. By using an appropriate refinement, it is possible to more easily identify a function's critical point, by using the following algorithm:

Let $X_{1} ; X_{2}, \ldots, X_{n}$ be subintervals of $X$ :

1. $F u b=\min \left[F\left(\operatorname{mid}\left(X_{1}\right)\right), F\left(\operatorname{mid}\left(X_{2}\right)\right), \ldots, F\left(\operatorname{mid}\left(X_{n}\right)\right), F u b\right]$

2. If $\operatorname{Inf}\left(F\left(X_{a}\right)\right)>F u b \Rightarrow$ Discard $X_{a} ;$ where $a=1,2, \ldots, n$

\section{Bisect every non - discarded intervals}

4. If width $\left(F\left(X_{a}\right)\right)>$ epsilon $\Rightarrow$ Go to 1

\section{Otherwise $\Rightarrow$ Finish}

Due to $X$ being a vector of intervals, the component with the higher width will be bisected. However, this only works for a Lipschitz function over the interval $X_{0}$, which has the property of isotonic inclusion and which also is a function of the type $F C_{n}\left(X_{0}\right)$ (Moore, Baker, and Cloud, 2009).

\subsection{Particle Swarm Optimization}

Particle Swarm Optimization (PSO) was proposed by Kennedy and Eberhart (1995). This method has been widely used to solve engineering problems, due to its high convergence speed, its relative high precision and its simplicity. It is based on the selection of a particle swarm (candidate solutions), over an Ndimensions sample space. These particles communicate to establish the best position found, related to an objective function. Each particle has a velocity and a position, which are updated according to two optimum values. These are, pbest and gbest, which refer to the coordinate where the best solution has been found, for a given particle and for the swarm, respectively. The velocity is updated by:

$$
v_{n}=\omega v_{n}+c 1 r \text { and }()\left(\text { pbest }_{n}-x_{n}\right)+c 2 r \text { and }()\left(\text { gbest }_{n}-x_{n}\right)
$$

Where $v_{n}$ is the $\mathrm{n}$-th particle's velocity; $x_{n}$ is the $\mathrm{n}$-th particle's position; $c 1$ is a self-trust factor; $c 2$ is the global trust factor; $\omega$ is a particle's inertia, and 
ran $\mathrm{d}(\mathrm{)})$ is a random number (between 0 and 1 ). After updating the velocity, a new position is found by multiplying it by a time period, $\Delta t$, i.e.:

$$
x_{n}=x_{n}+\text { Dt } v_{n}
$$

Generally, a time step of 1 is chosen. The iteration process can be stopped in several ways, being the top three: the definition of a maximum number of iterations, the definition of an expected value for the objective function and the definition of a minimum standard deviation in the particles' position.

\section{Process Description}

The main goal of this paper is to design a three-layered electromagnetic absorber (three thicknesses and three materials), using the optimization tools described above. The objective function is specified in equation (11).

\subsection{Software and Hardware}

All the programs of this research were run in a computer with the following specifications: Intel® Core $^{\mathrm{TM}} 2$ Quad Q8200 (4M Cache, $2.33 \mathrm{GHz}, 1333$ $\mathrm{MHz}$ FSB), with 2 GB RAM, and a Windows 7 Ultimate - 32 bits OS. It was programmed on MATLAB 7.2, and made use of Prof. Dr. Siegfried M. Rump's toolbox, INTLAB.

\subsection{Definition of the Work Band}

An exemplary absorber, to operate in the wireless technology range, and composed of three layers was designed. This working band comprises the mobile phone companies, Bluetooth communications protocol $(2.4 \mathrm{GHz})$ [IEEE 802.15.1], WiFi $(2,4 \mathrm{GHz}, 5 \mathrm{GHz}, 5,4 \mathrm{GHz})$ [IEEE 802.11a, IEEE 802.11b, IEEE 802.11g, IEEE 802.11n] and the ZigBee communication protocol $(0,868$ $\mathrm{GHz}, 0,915 \mathrm{GHz}, 2,4 \mathrm{GHz}$ ) [IEEE 802.15.4], among others.

\subsection{PSO Implementation}

After some preliminary tests, the experimental conditions for the absorber design, such as a swarm with 30 particles, whose velocity is inverted when trying to leave the search space, were defined. Also, the original non-modified form of PSO's algorithm is used. The layer thickness was set between 0.1 and $2.0 \mathrm{~mm}$, since a too thick/thin layer cannot be justified for practical applications. For running the optimization, a materials bank was defined, with specific permittivity and 
permeability, as shown in Table 1 . The material for each layer is selected from the 16 available. For the implemented swarm, $\omega$ was defined to decrease from 0.9 to 0.4 during the optimization process. Constants $c_{1}$ and $c_{2}$ are assumed to be 1.46 . Besides, gbest is determined after each particle has been evaluated on the objective function and through comparison with the previous one. Velocity is defined in a similar range than position. For thickness, it was defined between -0.0021 and 0.0021 , and for the materials, it was defined between -16.1 and 16.1.

Table 1. Materials Bank

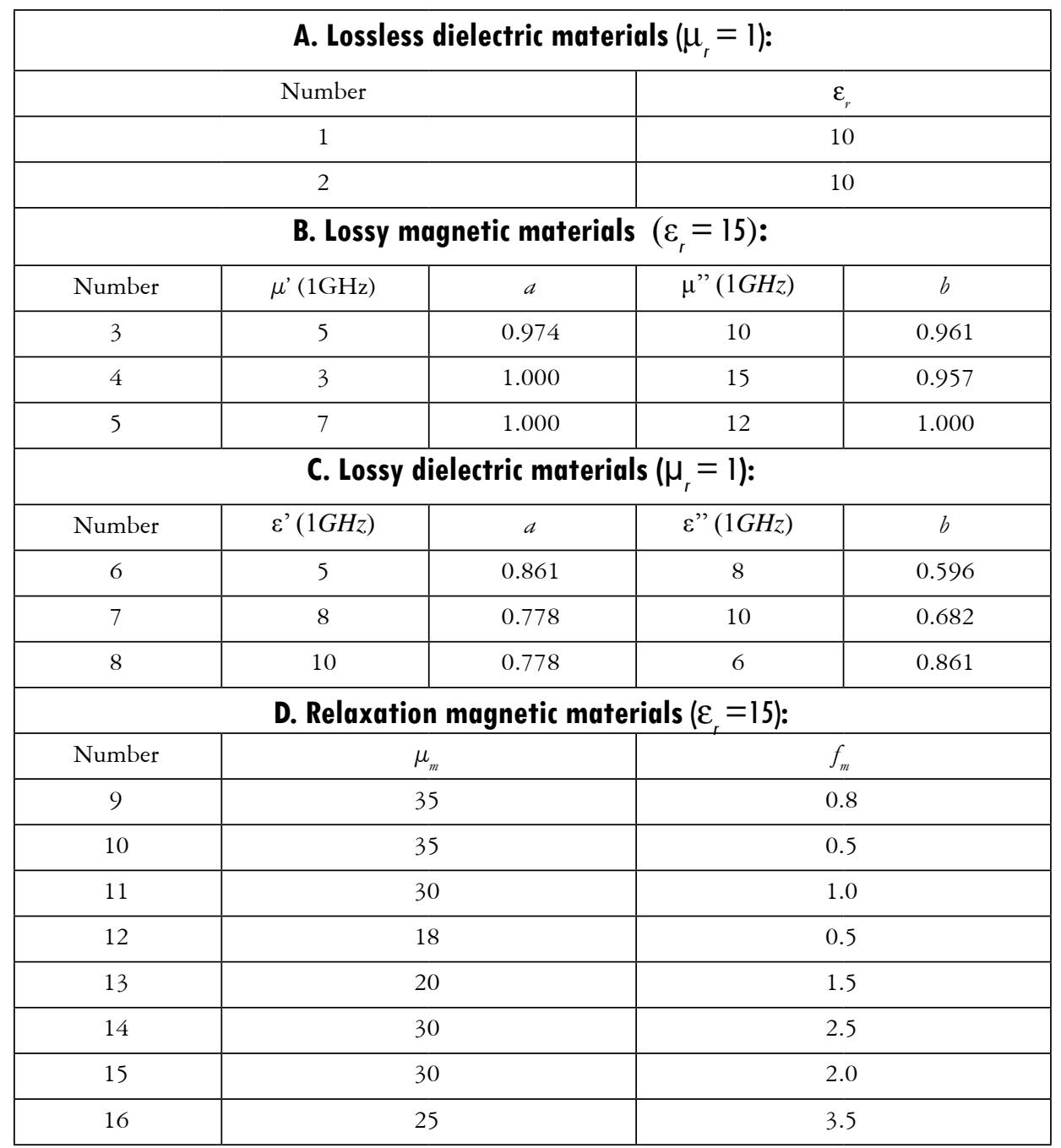

Source: Robinson and Rahmat-Samii (2004). 


\subsection{Implementation of the Algorithm Based on Intervals}

At the beginning, the algorithm was run for 19 hours, with a refinement of 6 and for some given materials (number 16,3 and 4, in that order), and it was not possible to achieve conclusive results. Because of this low algorithm convergence speed, it was necessary to implement an extra method, which was:

1. $\left[X_{1}, X_{2}\right]=\operatorname{Bisection}(X)$

2. $\left[K_{1}, K_{2}, \ldots, K \psi\right]=\operatorname{Bisection}\left(X_{1}\right) \psi$ times $\left[\left[U_{1}, U_{2}, \ldots, U_{\psi}\right]=\right.$ Bisection $\left(X_{2}\right) \psi$ times

3. $\min \left(X_{1}\right)=\min \left(F\left(\operatorname{mid}\left(K_{1}\right)\right), F\left(\operatorname{mid}\left(K_{2}\right)\right), \ldots, F\left(\operatorname{mid}\left(K_{\psi}\right)\right)\right)$

4. $\min \left(X_{2}\right)=\min \left(F\left(\operatorname{mid}\left(U_{1}\right)\right), F\left(\operatorname{mid}\left(U_{2}\right)\right), \ldots, F\left(\operatorname{mid}\left(U_{\psi}\right)\right)\right)$

5. If $\min \left(X_{1}\right)=\min \left(X_{2}\right) \Rightarrow$ Increase $n$ and go to (2)

6. If $\min \left(X_{1}\right)<\min \left(X_{2}\right) \Rightarrow X=X_{2}$

a. Otherwise $\Rightarrow X=X_{1}$

\section{Go to (1)}

This algorithm reduces the initial solution space, with a low chance of discarding the real solution space. This probability is inversely proportional to the number of divisions $\psi$ on each space.

\section{Results}

\subsection{Results Achieved Using PSO}

The PSO algorithm was run 30 times in order to get the optimal design over the frequency band $[0.2 \mathrm{GHz}-6 \mathrm{GHz}$. Nevertheless, three different possible designs were found during this process, as shown in Table 2. The behavior of these three absorbers is shown in Figure 2. 
Table 2. Designs Using PSO

\begin{tabular}{|l|c|c|c|c|c|c|}
\cline { 2 - 7 } \multicolumn{1}{c|}{} & \multicolumn{2}{c|}{ First Design } & \multicolumn{2}{c|}{ Second Design } & \multicolumn{2}{c|}{ Third Design } \\
\cline { 2 - 7 } \multicolumn{1}{c|}{} & Material & $\begin{array}{c}\text { Thickness } \\
{[\mathrm{mm}]}\end{array}$ & Material & $\begin{array}{c}\text { Thickness } \\
{[\mathrm{mm}]}\end{array}$ & Material & $\begin{array}{c}\text { Thickness } \\
{[\mathrm{mm}]}\end{array}$ \\
\hline Layer 1 & 16 & 0.5998 & 16 & 0.6842 & 16 & 0.5845 \\
\hline Layer 2 & 3 & 2.0000 & 12 & 1.8003 & 5 & 1.7433 \\
\hline Layer 3 & 4 & 1.6268 & 4 & 1.6890 & 4 & 1.9588 \\
\hline
\end{tabular}

Source: Authors' own presentation.

Figure 2. Behavior of the Absorbers over the Frequency Band for the Three Designs
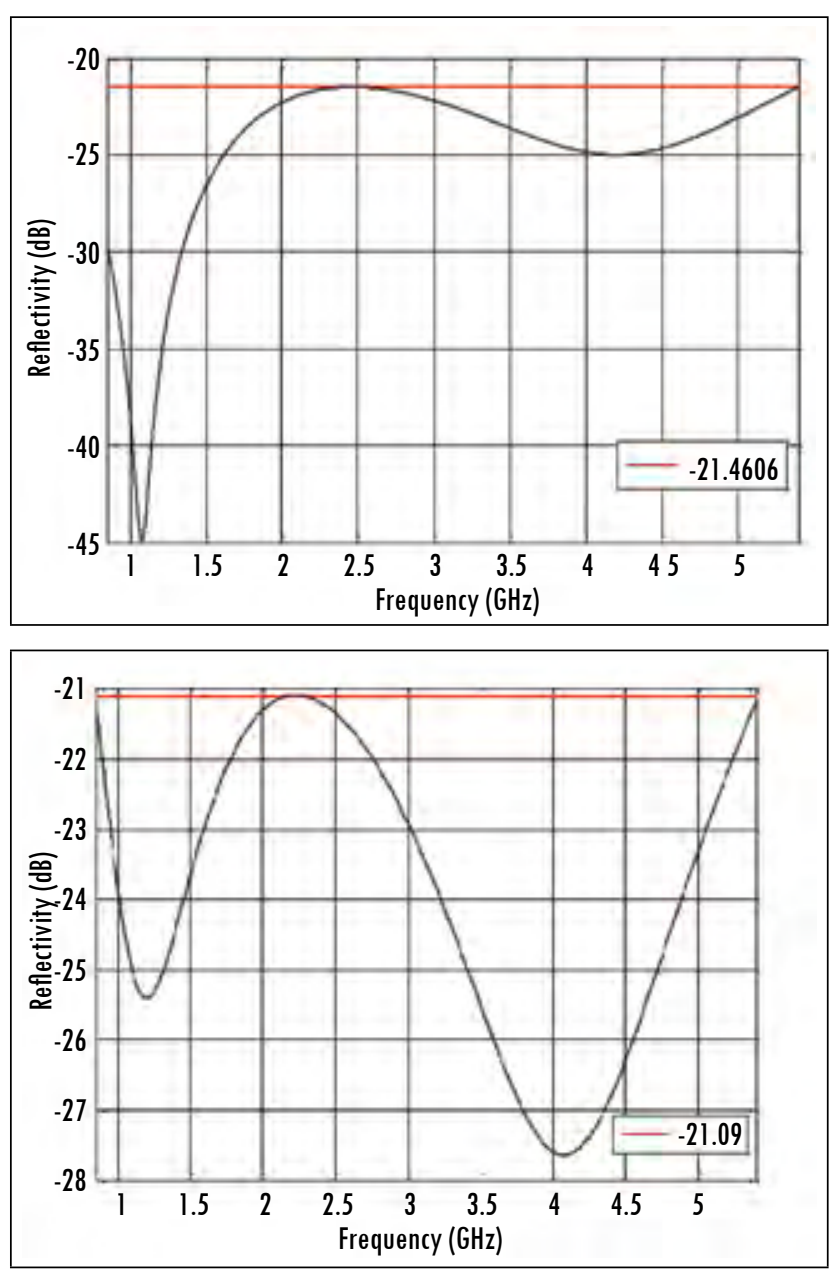

Ing. Univ. Bogotá (Colombia), 18 (1): 27-42, enero-junio de 2014 


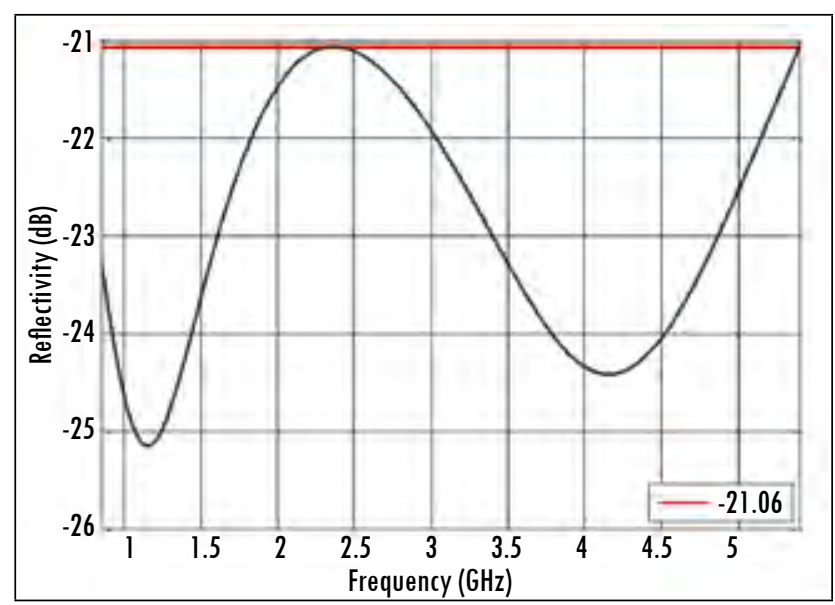

Source: Authors' own presentation.

It is important to remark the horizontal line on the plots. From these three designs, the first one is the best, since it has a lower critical point $(-21,4606 \mathrm{~dB})$, even though all of the optimum designs were in the range $[-21,5000,-20,9166]$. After gathering all the information, it was possible to determine the algorithm accuracy. Out of 30 runs, 23 were inside the error margin $\left(1 \times 10^{-5}\right)$, and were considered as successful (event A). It was then possible to obtain the probability of achieving the optimum design, and the probability of achieving a nonoptimum point (event B), i.e.:

$$
P(A)=\frac{23}{30} * 100 \approx 76,667 \% \text { and } P(B)=1-P(A) \approx 23,333 \%
$$

Using conditional probability, it was possible to establish the minimum number of runs (n), required for having a high probability of finding the optimum design (set at 99.9\%). Since the execution of the algorithm (event $\mathrm{C}$ ) is an independent event:

$$
\begin{gathered}
P\left(C_{1} \cap C_{2}\right)=P\left(C_{1}\right) P\left(C_{1} \mid C_{2}\right)=P(C 1) * P\left(C_{2}\right), \text { so } \\
\frac{99,9}{100} \leq 1-(0,23333)^{n}
\end{gathered}
$$

After solving, it can be found that $n \geq 4,7$, so $n \approx 5$. 


\subsection{Results Achieved Using the Algorithm Based on Interval Analysis}

In order to define the optimum materials for the absorber, the algorithm to reduce the solution space was implemented, running all possible combinations of materials. The number of divisions was set to 15 , and the best materials were found to be the same provided by PSO (numbers 16, 3 and 4 in that order). Striving to reduce convergence time even further, the number of divisions was varied from 15 to 10 , finding that $\psi=11$ was the lowest value that yielded correct results. Under this condition, a convergence time was of 31488.4691 seconds (8.7468 hours) was achieved. Having defined the materials, the same algorithm was used to reduce the solution space. In order to evaluate the influence of such algorithm in the results, the cases for 12 and 15 runs were studied. The difference between this two, is the size of initial solution space. Results are shown in Table 3. The final absorber, for both cases, fits well with the one designed by PSO, however, it requires considerably more time for convergence.

Table 3. Results Using Interval Analysis

\begin{tabular}{|l|l|l|}
\cline { 2 - 3 } \multicolumn{1}{c|}{} & \multicolumn{1}{c|}{ Case I } & \multicolumn{1}{c|}{ Case II } \\
\hline Initial interval & {$[0.000574999,0,000693750]$} & {$[0.000574999,0.000634375]$} \\
(recalculated) & {$[0.001881250,0,002000000]$} & {$[0.001940625,0.002000000]$} \\
& {$[0.001524999,0,001643750]$} & {$[0.001584375,0.001644375]$} \\
\hline Minimum $(\mathrm{dB})$ & -21.4605 & -21.4605 \\
\hline Convergence time $(\mathrm{s})$ & 4196.2323 & 1620.1082 \\
\hline Layer thicknesses $(\mathrm{mm})$ & $0.6009 ; 1.9963 ; 1.6326$ & $0.6009 ; 1.9963 ; 1.6326$ \\
\hline
\end{tabular}

Source: Authors' own presentation.

\section{Conclusions}

The final design of the electromagnetic absorber exhibited good performance for the frequency band $(0.85 \mathrm{GHz}$ to $5.4 \mathrm{GHz})$. An appropriate selection of the most relevant parameters and conditions, such as the swarm size and the boundary criteria, increases the likelihood of achieving a correct answer with a reduced number of runs when using PSO. On the other hand, the design through interval analysis requires a considerable amount of computer resources and several approximations for identifying the most appropriate material and for reducing the initial solution space. Nevertheless, since this is a deterministic method, the identification of the optimum parameters is carried out in one execution. Besides, the success of the proposed interval method depends on an 
appropriate selection of $\psi$. For this problem, it requires to be equal or greater than 11 to guarantee convergence to a correct value. After comparing both methods, it can be concluded that PSO performs better than interval analysis. The former requires about five minutes identifying the optimum design, with a success probability of $99.9 \%$. The latter, on the other hand, required about nine hours detecting it, even after the implementation of a time reducing method. This is mainly due to the complexity of the objective function, which generates an overestimate for relatively small input intervals. For a following research, it is intended to develop a hybrid method with both approaches, striving to achieve a balance between precision, reproducibility and computation time. According to Liu et al. (2009), where a five layers absorber was designed, it can be affirmed that results obtained are highly reliable. In such paper, the critical point (i.e. maximum reflectivity over the frequency band) is around $-21 \mathrm{~dB}$; the proposed optimal design reached a critical point of $-21.46 \mathrm{~dB}$ using just three layers, which represents a considerable gain.

\section{References}

AFSAR, M. et al. A millimeter-wave tunable electromagnetic absorber based on e-Al- $\mathrm{Fe}_{2} \mathrm{O}_{3}$ nanomagnets. IEEE Transactions on Magnetics. 2011, vol. 47, no. 2, pp. 333-336.

BRONWELL, A. Transmission-line analogies of plane electromagnetic-wave reflections. Proceedings of IRE. 1994, vol. 32, no. 4, pp. 233-241.

CHAMAANI, S. et al. Multi-objective particle swarm optimization for electromagnetic absorber design. Progress in Electromagnetics Research (PIER). 2008, vol. 79, pp. 353-366.

CUI, S. WEILE, D.S. and VOLAKIS, J.L. Novel planar electromagnetic absorbers design using genetic algorithm. IEEE Transactions on Antennas and Propagation. 2006, vol. 54, no. 6, pp. 1811-1817.

CUI, S. and WEILE, D. S. Application of a novel parallel swarm optimization to design electromagnetic absorber. Antennas and Propagation Society International Symposium, IEEE. 2005, pp. 3616-3624.

KENNEDY, J. and EBERHART, R.C. Particle swarm optimization. Proceedings of IEEE Con, Neural Networks IV, Piscataway, NJ, 1995, pp. 1942-1948.

LIU, H. et al. Electromagnetic wave absorber optimal design based on improved particle swarm optimization. EMC'2009, IEICE, Kyoto, Japan, 2009, pp. 797-800.

MICHIELSSEN, E. et al. Design of lightweight, broad-band microwave absorbers using genetic algorithms. IEEE Transactions on Microwave Theory and Techniques. 1993, vol. 41, no. 617, pp. 1024-1031.

MOORE, R.E., BAKER, R. M. and CLOUD, M.J. Introduction to interval analysis. Philadelphia: Society for Industrial and Applied Mathematics, 2009. 
NORNIKMAN H. et al. Study and simulation of an edge couple Split ring resonator (EC-SRR) on truncated pyramidal microwave absorber. Progress in Electromagnetics Research. 2012, vol. 127, pp. 319-334.

ROBINSON, J. and RAHMAT-SAMII, Y. Particle swarm optimization in electromagnetics. IEEE Transaction on Antennas and Propagation. 2004, pp. 397-407.

VECCHIA, P. et al. Exposure to high frequency electromagnetic fields, biological effects and health consequences (100 kHz-300 GHz). ICNIRP 16, 2009.

WATTS C. et al. Metamaterial electromagnetic wave absorbers. Verlag: Wiley-VCH, 2012.

ZHAO, B. et al. Fabrication and electromagnetic characteristics of microwave absorbers containing $\mathrm{Li}_{0.35} \mathrm{Zn}_{0.3} \mathrm{Fe}_{2.35} \mathrm{O}_{4}$ micro-belts and nickel-coated carbon fibers. Journal of Magnetism and Magnetic Materials. 2013, vol. 345, pp. 249-254. 